Atmos. Chem. Phys. Discuss., 9, 3535-3553, 2009

www.atmos-chem-phys-discuss.net/9/3535/2009/

(C) Author(s) 2009. This work is distributed under

the Creative Commons Attribution 3.0 License.

This discussion paper is/has been under review for the journal Atmospheric Chemistry and Physics (ACP). Please refer to the corresponding final paper in $A C P$ if available.

\title{
Deep-convective vertical transport: what is mass flux?
}

\section{J.-I. Yano}

CNRM-GAME, Meteo France and CNRS, Toulouse, France

Received: 18 November 2008 - Accepted: 8 January 2009 - Published: 3 February 2009

Correspondence to: Jun-Ichi Yano (jun-ichi.yano@meteo.fr)

Published by Copernicus Publications on behalf of the European Geosciences Union.

\section{ACPD}

9, 3535-3553, 2009

What is mass flux?

J.-I. Yano

\section{Title Page}

Abstract

Conclusions

Tables

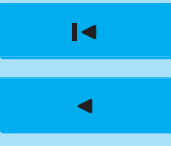

Back

Full Screen / Esc

Printer-friendly Version

Interactive Discussion 


\section{Abstract}

Conceptual basis for the convective mass flux that is used in parameterizations is reviewed from a historical perspective. The main idea of the convective mass flux formulation does not purely reside in dividing the grid-box averaged vertical velocity into 5 several mass flux components such as convective updrafts, downdrafts, and environmental subsidence. The main point rather resides on assuming different vertical profiles for transported quantities for different components. From this point of view, the best way to turn off the convective transport of chemical species is to set the vertical profile of chemical species within convective components (both updrafts and downdrafts) equal to that of the environment. This procedure turns out to be equivalent of simply turning off a standard convective chemical transport package.

\section{Introduction}

Deep convection is a very efficient agent for transporting various physical quantities vertically in the atmosphere; especially over the tropics, not only heat (dry entropy) etc (Yano et al., 2003).

In the majority of current convective parameterizations, these deep-convective transport processes are represented by a quantity called "mass flux". However, the understanding of this quantity in the convective parameterization community has a peculiar status. Listening to a story from a certain workshop on convection by A. Grant (personal communication, 2004), I had to conclude that what St. Augustine said in his "Confession" on time exactly applies for mass flux in the convective parameterization community: "Everyone believes they know what mass flux is, but as soon as they are asked what it is, they immediately realize they do not know what the mass flux is." (The

The confusion further deepens when the concept is extended into a context of chem-

9, 3535-3553, 2009

What is mass flux?

J.-I. Yano

Title Page

Abstract

Conclusions

Tables

Figures

14

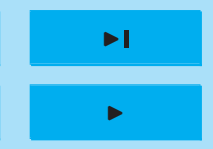

$\triangleleft$

Back

Close

Full Screen / Esc

Printer-friendly Version

Interactive Discussion 
ical transports. The goal of the present paper is to try to make a point clear that mass flux is a more concrete and elementary concept than time, and point out implications in the context of convective chemical transport.

A direct motivation of the present paper is provided by Lawrence and Salzmann 5 (2008), who exactly asked this question more concretely in quantifying the contribution of convective transport in global chemistry budget. The present paper, in turn, intends to point out an aspect that is less emphasised by Lawrence and Salzmann (2008).

Thus, the main intension of the present contribution is to clarify the concept of mass flux in the context of convective transport. The simplest possible examples are adopted 10 for demonstrative purposes. Discussions on technical issues are kept minimum, although all these are important in actually developing a mass flux based scheme.

\section{Tube Mechanism: hot tower hypothesis by Riehl and Malkus (1958)}

In order to understand well what mass flux is, it is best try to trace back a historical root for a concept of the convective cumulus parameterization. I believe it is not misleading 15 to state that a landmark work by Riehl and Malkus (1958) (see also their re-visit a decade later: Riehl and Simpson, 1979) pointed us to a need for a parameterization of deep cumulus convection in the tropical mean circulation. The paper examined the moist-static energy budget of the tropical mean circulation, namely the Hadley-Walker circulation in the contemporary notion, although clearly they were not aware of the Walker circulation.

Here we reproduce their original theoretical argument by replacing the moist static energy by the moist entropy. The moist entropy $S_{m}$, related to the equivalent potential temperature $\theta_{e}$ by

$S_{m}=C_{p} \ln \theta_{e}$

25 is a thermodynamic quantity conserved when a given air particle does not receive any heat from an exterior (such as radiative heating and surface fluxes). Here, $C_{p}$

What is mass flux?

J.-I. Yano

Title Page

Abstract

Conclusions

Tables

14

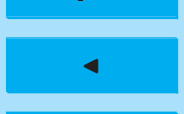

Back

Introduction

References

Figures

$>1$

$\rightarrow$

Close

Full Screen / Esc

Printer-friendly Version

Interactive Discussion 
is the heat capacity of moist air at constant pressure, and the equivalent potential temperature is given by

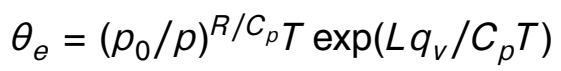

9, 3535-3553, 2009

to good approximation with $p$ the pressure, $p_{0}=1000 \mathrm{hPa}$ the reference pressure, $R$ 5 the gas constant for the air, $T$ the temperature, $L$ the latent heat of condensation, and $q_{v}$ the mixing ratio of the water vapor. The moist entropy furthermore approximately reduces to the moist static energy when a hydrostatic balance is satisfied.

Their main theoretical argument simply hinges on examining a tropical vertical profile of moist entropy in the tropical atmosphere, which is typically highest at the surface, decreases with height towards a middle troposphere, then begin to increase with height, as schematically shown by a solid curve in Fig. 1.

Riehl and Malkus (1958) considered the heat budget over an ascending branch of the Hadley circulation. The moist entropy is supplied from the surface by sensible heat flux and evaporation, and then lost to space by radiation dominantly at the tropopause level. Additionally, the strongest export of the moist entropy happens at an upper level of the troposphere associated with a divergence branch of the Hadley circulation. Note that the moist entropy is conserved even under condensation processes. ${ }^{1}$ Then the main question to ask is: how the moist entropy is transported upwards all the way through the troposphere from the surface to the tropopause level so that the budget can be closed?

A simple physical consideration immediately tells us that the moist-entropy budget cannot be closed by simply considering a "large scale" ascent $\bar{W}$ in the ascending branch of the Hadley-Walker circulation. Here, by "large scale", we refer to type of circulations that can readily defined by a convectional radiosonde network with, say, a $1000-\mathrm{km}$ resolution.

\footnotetext{
${ }^{1}$ More precisely, as far as ice fusion effects are negligible. That is a good leading-order approximation.
}

What is mass flux?

J.-I. Yano

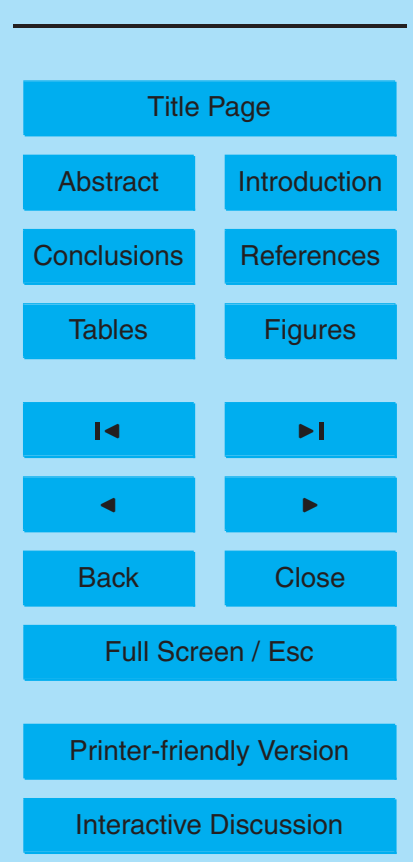

Interactive Discussion

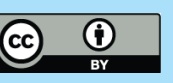


More precisely, Riehl and Malkus (1958) considered the climatology over the ascending region of the Hadley-Walker circulation (equatorial trough zone). Probably, this original interpretation on $\bar{w}$ makes it easier to understand the following argument, though a generalisation of the notion of "large-scale" is relatively straightforward, as 5 will be re-addressed in the next section.

Such a large-scale ascent modifies the moist entropy $\bar{S}_{m}$ with a rate:

$-\bar{w}\left(\frac{d}{d z} \bar{S}_{m}\right)$

The formula shows that the transport of moist entropy is always the direction of down slope of its vertical profile. Hence, under an ascending motion, the moist entropy is transported upwards in a lower half of the troposphere and downwards in an upper half of the troposphere, by following the given vertical profile of the moist entropy schematically shown in Fig. 1. The obtained vertical advection rate is schematically shown by a solid curve in Fig. 2. Importantly, the argument here is valid as far as the air is ascending all the way through the troposphere without depending on a detail of the vertical 15 profile.

Consequently, under the given large-scale circulation, the moist entropy cannot be transported upwards all the way through the troposphere, but it is accumulated to a middle troposphere, thus the moist-entropy budget is not closed. In other words, in order to accomplish an upward transport of moist entropy all the way through the troposphere, a downward mean motion must be introduced in an upper half of the troposphere, which is not consistent with the observed structure of the Hadley circulation. Hence, something else must be going on.

Riehl and Malkus (1958) proposed that a way to avoid this dilemma is to assume an existence of tube-like entities penetrating through the whole troposphere. Within these "tubes", the surface moist entropy is more or less directly transported to the tropopause level without mixing with the air outside the "tubes". In order that such a process to be realized, the vertical transport within the "tubes" must be fast enough so that the surface air particle can reach the tropopause without substantial mixing

What is mass flux?
J.-I. Yano

Title Page

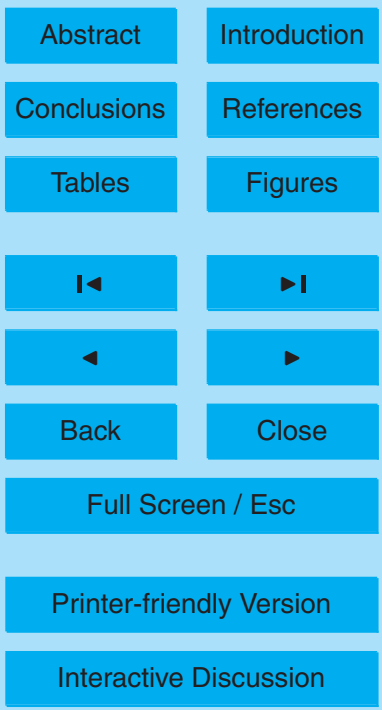

Interactive Discussion 
with the surrounding air, as indicated by a dashed line in Fig. 1. The resulting vertical advection rate is schematically shown by a dashed curve in Fig. 2: it provides a sharp sink and source at the surface and the tropopause levels, respectively (dashed curve) as shown by Eq. (11) below. Those sink and source balance with the surface flux and 5 radiative cooling, respectively, thus the entropy budget is closed.

These "tubes" must be tall (rather than fat) so that their relative isolation is well insured. These tube-like entitles are subsequently named the "hot towers" ${ }^{2}$, phenomenologically corresponding to the deep convective towers of few kilometer scales in the contemporary terminology.

Few more remarks should follow: the proposed deep-convective transport process is fast and efficient, but only locally. A fractional area occupied by these convective towers ("hot towers") within the whole ascending branch of the Hadley-Walker cell is relatively small, thus an integrated effect of the transport by convective towers averaged over the whole Hadley-Walker cell is not at all faster than the one realized by the large15 scale mean transport in the order of magnitude. The point is well summarized by Fig. 4 of Lawrence and Salzmann (2008), which shows both tropical-averaged total and convective vertical mass fluxes. The main effect by introducing "hot towers" into the Hadley-Walker circulation is to create a transported quantity well-preserved against lateral mixing with environment, as schematically represented by a dashed line in Fig. 1 against an environmental profile (solid curve). As a result, the direction of the vertical moist-entropy flux is changed as summarized in Fig. 2.

\footnotetext{
${ }^{2}$ Note that the word does not appear in the original paper, though it is used in their re-visit (Riehl and Simpson, 1979). I would like to thank E. Zipser for pointing out to me this fact.
}

What is mass flux?

J.-I. Yano

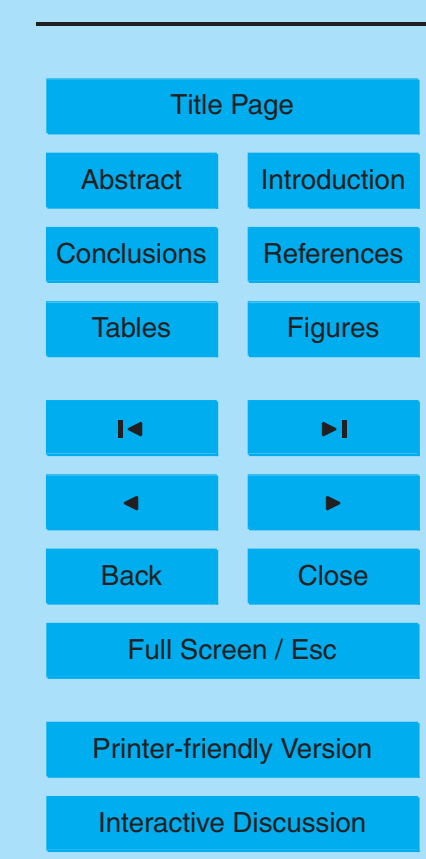

Interactive Discussion 


\section{Basis of the mass flux formulation}

\subsection{Under a framework of Riehl and Malkus}

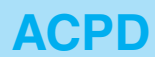

Now we introduce a basic notion of the mass flux based description for the convective vertical transport by elaborating the idea of convective "tubes" introduced by Riehl 5 and Malkus (1958). In this perspective, the basic idea of Riehl and Malkus (1958) is to divide the mean vertical motion $\bar{w}$ averaged over an ascending branch of the Hadley-Walker circulation into the two components: the convective updraft $M_{c}$ and the environmental descent $M_{e}{ }^{3}$

The convective transport may, in principle, be decomposed into a spectrum of plumes 10 as formulated by Arakawa and Schubert (1974), but we consider only a single component, corresponding to what called the bulk mass flux formulation, for the sake of simplicity. Note that the majority of current convective parameterizations use the bulk formulation. Subsequent studies (Zipser, 1969, 1977) showed that downdraft $M_{d}$ both in convective and meso-scales contribute significantly for vertical transports, thus we 15 may also add this effect into a decomposition of the vertical motion.

Consequently, the climatological-mean ascent $\bar{w}$ within a Hadley-Walker cell may be decomposed into the three components:

$\bar{w}=M_{c}+M_{d}+M_{e}$.

Here, the quantity $M_{*}$ with the subscripts $*=c, d, e$ is called the mass flux. It can 20 be interpreted as multiplication of a typical vertical velocity, say, $w_{*}$ associated with a corresponding component designated by the subscript $*$, and a fractional area $\sigma_{*}$ occupied by a corresponding component, thus

$M_{*}=\sigma_{*} w_{*}$.

\footnotetext{
${ }^{3}$ In spite of its name the environment can also be ascending as shown by Lawrence and Salzmann (2008).
}

What is mass flux?

J.-I. Yano

Title Page

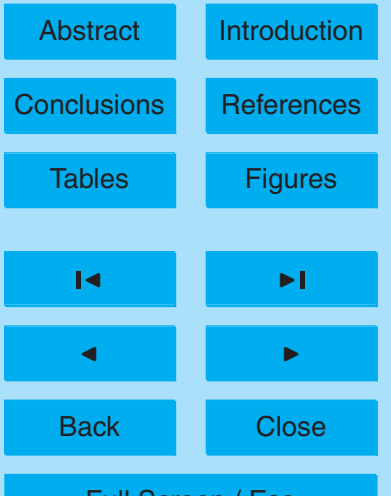

Full Screen / Esc

Printer-friendly Version

Interactive Discussion 
The basic idea here is to divide a horizontal area over the whole ascending branch of the Hadley-Walker circulation into three components (subdomains): convective updrafts, downdrafts, and the environment. Each occupies a fractional area $\sigma_{c}, \sigma_{d}, \sigma_{e}$, respectively, thus

$5 \sigma_{c}+\sigma_{d}+\sigma_{e}=1$

Within each component, we assume that the air moves vertically with a horizontally homogeneous velocity, $w_{c}, w_{d}, w_{e}$, respectively. The transport rate within each component is given by a multiplication of these two quantities, as given by Eq. (3) above.

The idea is schematically represented by Fig. 3. The areas for convective updrafts 10 and downdrafts are not necessarily confined to a single encircled subdomain, but more likely they are distributed all over the whole ascending region. As already emphasised in the last section, each updraft and downdraft element is well isolated within the ascending branch of the Hadley-Walker circulation. Note that the total fractional areas, by summing all these individual elements, are designated by $\sigma_{c}$ and $\sigma_{d}$, respectively. 15 The remaining area is considered as the environment, which is either descending or ascending. Here, we assume that values of physical variables are horizontally homogeneous for each subdomain, though each of them is actually separated into number of separate elements. ${ }^{4}$

As a result, a vertical transport rate ${ }^{5}$ of, say, moist entropy $S_{m}$ within each subdomian 20 is given, in flux form ${ }^{6}$, by

$\frac{\partial}{\partial z} M_{*} S_{m *}$

${ }^{4}$ Alternatively, if readers wish, all the physical variables may be considered as ensemble averages. In this case, however, additional assumptions would be required for deriving the following results.

${ }^{5}$ Unlike the last section, we do not add a minus sign in the following just for brevity.

${ }^{6}$ The flux form is adopted, in place of an advective form as in the last section, for an ease of deriving a final expression Eq. (11) given below.
ACPD

9, 3535-3553, 2009

What is mass flux?

J.-I. Yano

Title Page

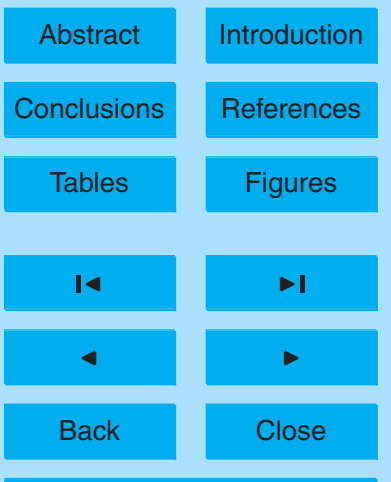

Full Screen / Esc

Printer-friendly Version

Interactive Discussion 
with $*=c, d, e$. By taking a sum of the contributions from all these subdomains, we obtain a total rate of vertical transport over the whole ascending branch of the HadleyWalker circulation as

$$
\frac{\partial}{\partial z} \overline{M S_{m}}=\frac{\partial}{\partial z} M_{c} S_{m c}+\frac{\partial}{\partial z} M_{d} S_{m d}+\frac{\partial}{\partial z} M_{e} S_{m e}
$$

5 where, as in Eq. (2), the overbar indicates climatological mean.

At this point, we should emphasize that the main reason for dividing the whole ascending branch of the Hadley-Walker circulation into three subdomains is not due to, for example, a convective vertical velocity $w_{c}$ much larger in magnitude than an environmental value $w_{e}$, but primarily due to qualitatively different vertical profiles $S_{m c}, S_{m d}$ for a transported variable within convective elements (both updrafts and downdrafts) compared to $S_{m e}$ in the environment. Such distinctively different vertical profiles for the environment and the convective updrafts are already schematically shown in Fig. 1.

In order to make this point in a totally trivial manner, let's consider an extreme situation that these three vertical profiles are identical, i.e.

$15 S_{m c}=S_{m d}=S_{m e}=\bar{S}_{m}$

Substitution of Eq. (7) into Eq. (6), with a help of Eq. (2), leads to

$$
\frac{\partial}{\partial z} \overline{M S_{m}}=\frac{\partial}{\partial z} M_{c} S_{m}+\frac{\partial}{\partial z} M_{d} S_{m}+\frac{\partial}{\partial z} M_{e} S_{m}=\frac{\partial}{\partial z} \bar{w} \bar{S}_{m}
$$

i.e. no convective transport effect is seen in a grid-box average, although a convective vertical velocity $w_{c}$ may locally be substantially larger in magnitude than an environmental value $w_{e}$.

Under a framework of Riehl and Malkus (1958) discussed in the last section, the moist entropy is assumed to be transported to a tropopause level, $z_{t}$, from a surface level (but not necessarily right at the surface, but at the top of the boundary layer, for example), $z_{s}$, without mixing. That implies a vertical profile constant with height:

${ }_{25} S_{m c}=\bar{S}\left(z_{S}\right)$.

What is mass flux?

J.-I. Yano

Title Page

Abstract

Introduction

Conclusions

Tables

References

Figures

14

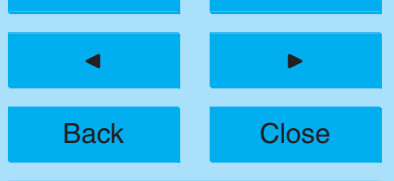

Full Screen / Esc

Printer-friendly Version

Interactive Discussion 
Recall that the vertical profile of the moist entropy for the convective-updraft component defined by Eq. (9) is shown by a dashed line in Fig. 1.

A convective mass flux is assumed constant throughout the updraft zone (i.e. $\left.z_{s}<z<z_{t}\right)$ :

${ }_{5} M_{c}= \begin{cases}0 & z \leq z_{s} \\ M_{c, 0}=\text { const. } & z_{s}<z<z_{t} \\ 0 & z \geq z_{t}\end{cases}$

where $z_{t}$ is the top of the convective updraft. As a result, the convective vertical transport is

$\frac{\partial}{\partial z} M_{c} S_{m c}=S_{m c} \frac{\partial}{\partial z} M_{c}=\mu S_{m c}\left[\delta\left(z-z_{s}\right)-\delta\left(z-z_{t}\right)\right]$,

where $\mu \equiv M_{c, 0}$ is the rate that moist entropy is entrained into the hot tower at $z=z_{s}$ and that it is detrained at $z=z_{t}$. Note that a character of the transport given by Eq. (11) is qualitatively different from the one for the environment as given by Eq. (1) as schematically indicted by a dashed line in Fig. 2.

\subsection{Generalisation}

In the last subsection, we have introduced the notion of mass flux under a framework of Riehl and Malkus (1958) into the whole ascending branch of the Hadley-Walker circulation. On the other hand, in implementing this mass-flux formulation as a convective parameterization into a global model, the same idea must be applied to individual grid boxes. In other words, a square area marked by solid lines in Fig. 3 must be re-interpreted as representing a single grid-box domain.

In order to still justify all the previous reasonings, individual convective towers must be well isolated within a given grid box. In other words, the size of individual convective towers must be much smaller than the size of the grid box. This principle is often referred as the scale separation.

What is mass flux?

J.-I. Yano

Title Page

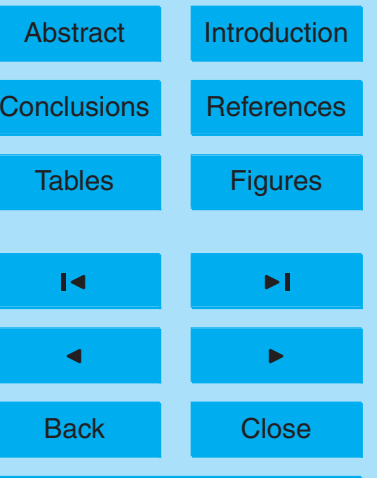

Full Screen / Esc

Printer-friendly Version

Interactive Discussion

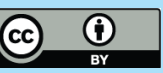


However, there is a more important implication of the scale-separation principle: all the quantities associated with the mass-flux formulation, i.e. $M_{*}$ and $S_{m *}$ with $*=c, d, e$, must be smooth functions of model "large-scale" variables. Especially, the convective variables such as $M_{c}$ and $S_{m c}$ should not change drastically from one grid-box to 5 next in order to ensure a smooth evolution of a global model under a given mass-flux parameterization.

There is still another point to make: the discussion of the last section is on the climatological mean state, thus no time dependence is considered. On the other hand, in a global modelling context, the system evolves with time, thus the decomposed variables 10 such as $M_{*}, S_{m *}$ must also evolve with time. Nevertheless, in standard implementations of mass-flux convective parameterization, analogous procedures as outlined by Eqs. (9-11) are employed every time step for diagnosing $M_{*}$ and $S_{m *}$ by assuming an instantaneous adjustment of convection to the environment (grid-box state). This hypothesis is called the convective quasi-equilibrium. An exact manner of posing a 15 quasi-equilibrium is, however, far from trivial. Such an exact procedure is called, at a more technical level, the closure problem, an important issue in actual implementation of a parameterization.

Hence there are a few subtle aspects of applying the mass-flux formulation into a global model by generalising the framework of Riehl and Malkus (1958). Nevertheless,

20 it is important to emphasize that the same principle as that of Riehl and Malkus is still applied to a model grid box in global modelling.

It may also be emphasized that a much more complex entrainment-detrainment structure than Eq. (11) is adopted in operational application. Nevertheless the basic argument of Riehl and Malkus (1958) should remain valid, and a profile of the moist entropy for the convective updraft should also remain overall homogeneous vertically, though some "erosion" inevitable happens by entrainment. In other words, in spite of the simplifications behind their theory, Riehl and Malkus (1958) provides an important pedagogical basis for understanding a basic concept of the mass flux.

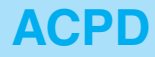

9, 3535-3553, 2009

What is mass flux?

J.-I. Yano

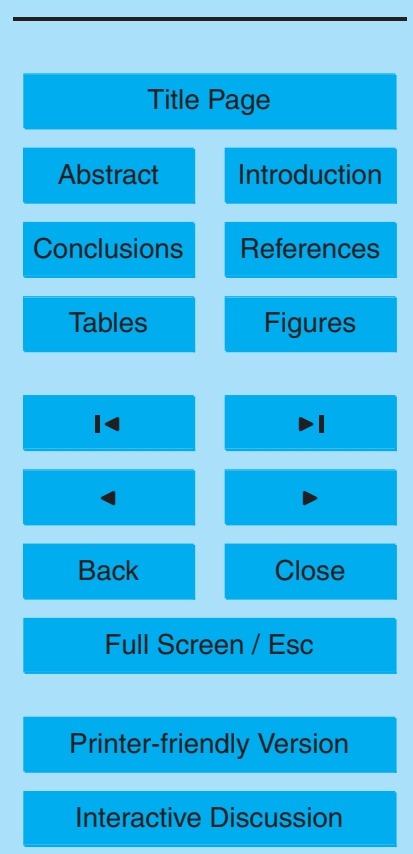

Interactive Discussion 


\section{How to turn off the convective chemical transport?}

\subsection{Conventional arguments}

Under a mass-flux convective representation, the convective chemical transport is handled in the same manner as the convective transport of moist entropy. Thus, replacing

$5 S_{m}$ by a mixing ratio $r$ of a chemical species in Eq. (6), the total vertical transport of a chemical species over a grid box is obtained:

$\frac{\partial}{\partial z} \overline{M r}=\frac{\partial}{\partial z} M_{c} r_{c}+\frac{\partial}{\partial z} M_{d} r_{d}+\frac{\partial}{\partial z} M_{e} r_{e}$

As emphasized by Lawrence and Salzmann (2008), in actual GCM implementations, the vertical transport is usually handled separately for a "grid-box mean" and for "con10 vection", respectively, thus

$\frac{\partial}{\partial z} \overline{M r}=\frac{\partial}{\partial z} \bar{w} \bar{r}+\left(\frac{\partial}{\partial z} M r\right)_{c}$

where the first term in the right hand side is the grid-box mean "large-scale" transport, the second term is the convective transport calculated by a convective parameterization package separately. The latter is defined by

${ }_{15}\left(\frac{\partial}{\partial z} \overline{M r}\right)_{c}=\frac{\partial}{\partial z} M_{c} r_{c}+\frac{\partial}{\partial z} M_{d} r_{d}+\frac{\partial}{\partial z} M_{e}^{\prime} r_{e}$

in which the last term contains residual mass flux defined by $M_{e}^{\prime} \equiv M_{e}-\bar{w}$. As emphasized by Lawrence and Salzmann (2008), it may be important to note that the sum of the mass fluxes considered within a convective parameterization package has no domain mean:

$20 M_{C}+M_{d}+M_{e}^{\prime}=0$.

We should clearly distinguish between Eq. (2) and (14). A similar point is made by Salzmann et al. (2004) in cloud-resolving modelling context.

What is mass flux?

J.-I. Yano

Title Page

Abstract

Introduction

Conclusions

References

Tables

Figures

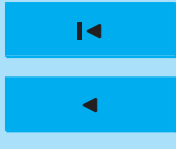

$\rightarrow 1$

Back

Close

Full Screen / Esc

Printer-friendly Version

Interactive Discussion 
Lawrence and Salzmann (2008) call $M_{e}^{\prime}$ the "convective subsidence", but probably it is better called the residual environmental subsidence, because it measures a part of environmental subsidence not counted by the grid-box averaged vertical motion $\bar{w}$. The two expressions Eqs. (12) and (13a, b) are equivalent under a standard asymptotic 5 limit $\sigma_{c} \sim \sigma_{d} \rightarrow 0$, because that leads to an approximation $r_{e} \simeq \bar{r}$. Note that the separation of the environmental subsidence $M_{e}$ into two components $\bar{w}$ and $M_{e}^{\prime}$ is totally artificial, though it is not without numerical consequences as emphasized by Lawrence and Salzmann (2008).

Under the given framework, there are two major ways for "turning off" the convective 10 transport, as extensively discussed by Lawrence and Salzmann (2008). The first is to simply turn off the convective transport package so that $(\partial \overline{M r} / \partial z)_{c}=0$. This is the standard procedure taken in the current sensitivity studies, but the choice is not free of problems. Most importantly, it turns off not only the convective transport processes $M_{c}$ and $M_{d}$ but also an associated residual environmental descent $M_{e}^{\prime}$, which should 15 in fact, be considered as a part of the total environmental descent $M_{e}=\bar{w}+M_{e}^{\prime}$. That is the main point emphasized by Lawrence and Salzmann (2008).

An alternative possibility is to more directly set the two components of mass flux associated with convection to zero, i.e. $M_{c}=M_{d}=0$. Such a procedure may be arguably unphysical, because by turning off two mass flux components, the mass continuity 20 Eq. (2) is no longer satisfied. Nevertheless, in spite of this serious defect, the second appears to be a more legitimate choice for estimating the convective effects in transport. Arguably, a break down of mass continuity is not a big deal, because we are artificially turning off a process which otherwise would exist, so we are distorting the physics any way. This very last point is of my own supplementing the major ar25

guments by Lawrence and Salzmann (2008). It should be emphasized that resulting distortions of physics would be minimum. As long as we set $M_{C}=M_{d}=0$ only for convective chemical transport keeping all the other physical processes in the model intact, conservation of air mass (but not including minor contributions by chemical species), heat, and moisture is ensured.

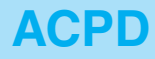

9, 3535-3553, 2009

What is mass flux?

J.-I. Yano

Title Page

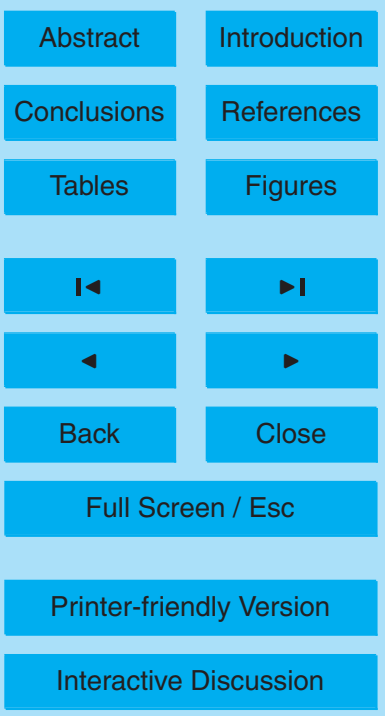

Interactive Discussion 


\subsection{A different perspective}

However, I would like to propose a very different perspective to this debate. The main point is to realize that we should not get confused with the word "transport" in order to turn off convective transport.

5 The main emphasis of Sects. 2 and 3 have been that we should not consider the basic idea of the mass flux decomposition as a procedure of simply dividing the gridbox mean vertical velocity $\bar{w}$ into various transport components (or mass fluxes) that transport a physical variable by different rates and even to different directions (e.g. convective updrafts vs. downdrafts).

10 Such a naive perspective totally misses the main point of the mass-flux idea: if the vertical mass fluxes are simply divided in few components, but if nothing else is modified, the total transport rate does not change, because by adding these transport components, regardless how fast locally it is, we simply recover the same total transport rate, as demonstrated by Eq. (8).

15 As we have seen from the historical review in Sect. 2, the convective transport is important in the tropical atmosphere, not because it is very fast (as already emphasized, we do not see this fastness in large-scale average), but because it transports the physical variables in a qualitatively different manner, by creating a "tube" like isolated structure, as schematically shown in Fig. 1. As a result, the transported quantity becomes different from that of the environment, as well distinguished by solid and dashed profiles in Fig. 1. In other words, what really matters in the mass flux transport is not the mass flux itself, but the vertical profiles $r_{c}$ and $r_{d}$ of the transported chemical species within convective updraft and downdraft.

Especially, when a time-scale associated with chemical reactions for a given chemical species is much longer than those associated with convection, it can be approximated as a conserved quantity (i.e. passive scalar) similar to the moist entropy. Then the profiles of $r_{c}$ and $r_{d}$ are much more homogeneous vertically than that of the environmental value $r_{e}$, exactly in the same manner as schematically shown for the moist

What is mass flux?

J.-I. Yano

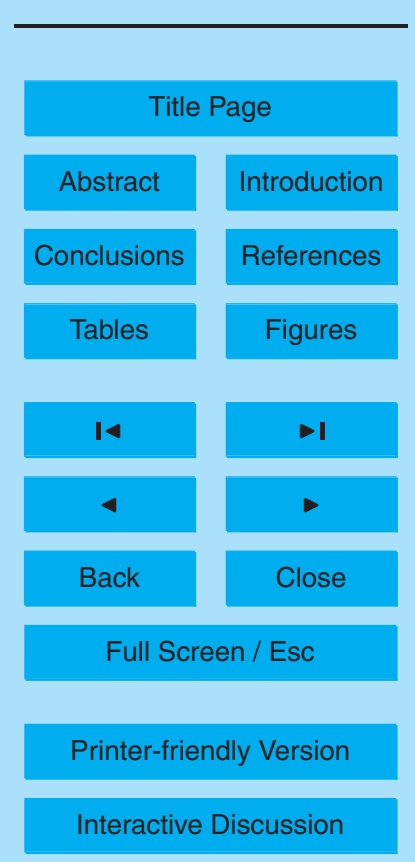

Interactive Discussion

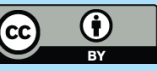


entropy in Fig. 1. These different vertical profiles provide qualitatively different transport characteristics between convection and environment (cf. Fig. 2).

From this perspective, the best way to turn off the convective chemical transport is not in turning off the convective mass fluxes (it furthermore distorts the mass continuity), 5 but to set the vertical profiles of a chemical species associated with the convective transport equal to that of the environment, i.e. $r_{c}=r_{d}=\bar{r}$. This turns out to be equivalent of turning off the convective transport package by setting $(\partial \overline{M r} / \partial z)_{c}=0$, but with a very different reasoning.

Unfortunately as a last word, we have to admit additional complexities associated with convective chemical transport: actual characteristics time-scale for chemistry is often much shorter than a time required for deep convection to transport an air particle from the surface to the tropopause so that the above assumption of a quasi-conserved passive scalar is not at all satisfied. Moreover, the chemistry becomes more involved because of the existence of precipitation processes. Especially, many chemical species 15 are washed out through precipitation. All these aspects must well be taken into account on top of the simplified arguments in considering the actual convective chemical transport processes. Nevertheless, it would still be revealing considering these complex convective chemical transport processes under a framework of Riehl and Malkus (1958) much closely.

20 Acknowledgements. Intensive discussions with Mark Lawrence, Marc Salzmann, Holger Tost for various occasions, led to the present manuscript, although finally we agree to disagree. Careful reading of the text by Clementine Junquas is much appreciated.

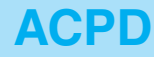

9, 3535-3553, 2009

What is mass flux?

J.-I. Yano

Title Page

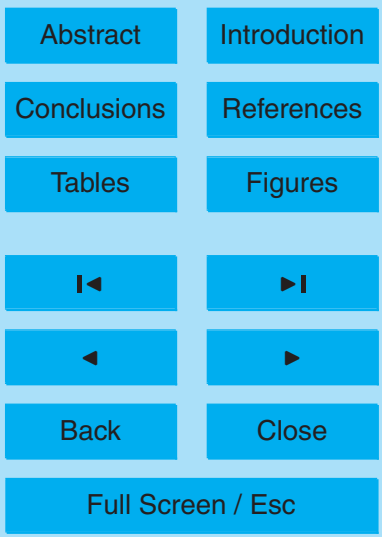

Printer-friendly Version

Interactive Discussion 


\section{References}

Arakawa, A. and Schubert, W. H.: Interaction of a cumulus cloud ensemble with the large-scale environment, Part I, J. Atmos. Sci., 31, 674-701, 1974. 3541

Lawrence, M. G. and Salzmann, M.: On interpreting studies of tracer transport by deep cumulus 5 convection and its effects on atmospheric chemistry, Atmos. Chem. Phys., 8, 6037-6050, 2008. 3537, 3540, 3541, 3546, 3547

Riehl, H. and Malkus, J. S.: On the heat balance in the equatorial trough zone, Geophysica, 6 , 503-538, 1958. 3537, 3538, 3539, 3541, 3543, 3544, 3545, 3549

Riehl, $\mathrm{H}$. and Simpson, J. M.: The heat balance of the equatorial trough zone, revisited, Contr. Atm. Phys., 52, 287-305, 1979. 3537, 3540

Salzmann, M., Lawrence, M. G., Phillips, V. T. J., and Donner, L. J.: Modelling tracer transport by a cumulus ensemble: lateral boundary conditions and large-scale ascent, Atmos. Chem. Phys., 4, 1797-1811, 2004. 3546

Yano, J. I., Donner, L. J., Yin, Y., Lawrence, M. G., Mari, C., and Stohl, A.: Meeting report: 15 Multidisciplinary discussions of convective chemical transport, EOS, 84, 327-330, 2003. 3536

Zipser, E. J.: The role of unsaturated convective downdrafts in the structure and rapid decay of an equatorial disturbance, J. Appl. Meteorol., 8, 799-814, 1969. 3541

Zipser, E. J.: Mesoscale and convective-scale downdrafts as distinct components of squall-line circulation, Mon. Weather Rev., 105, 1568-1589, 1977. 3541

9, 3535-3553, 2009

What is mass flux?

J.-I. Yano

Title Page

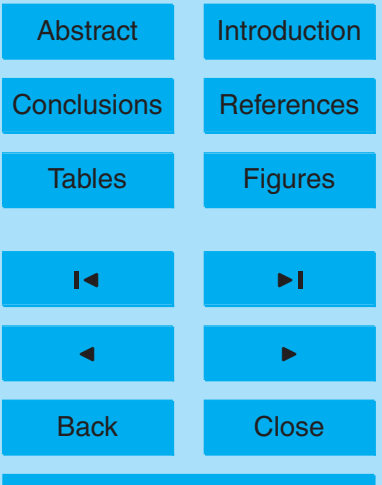

Full Screen / Esc

Printer-friendly Version

Interactive Discussion 


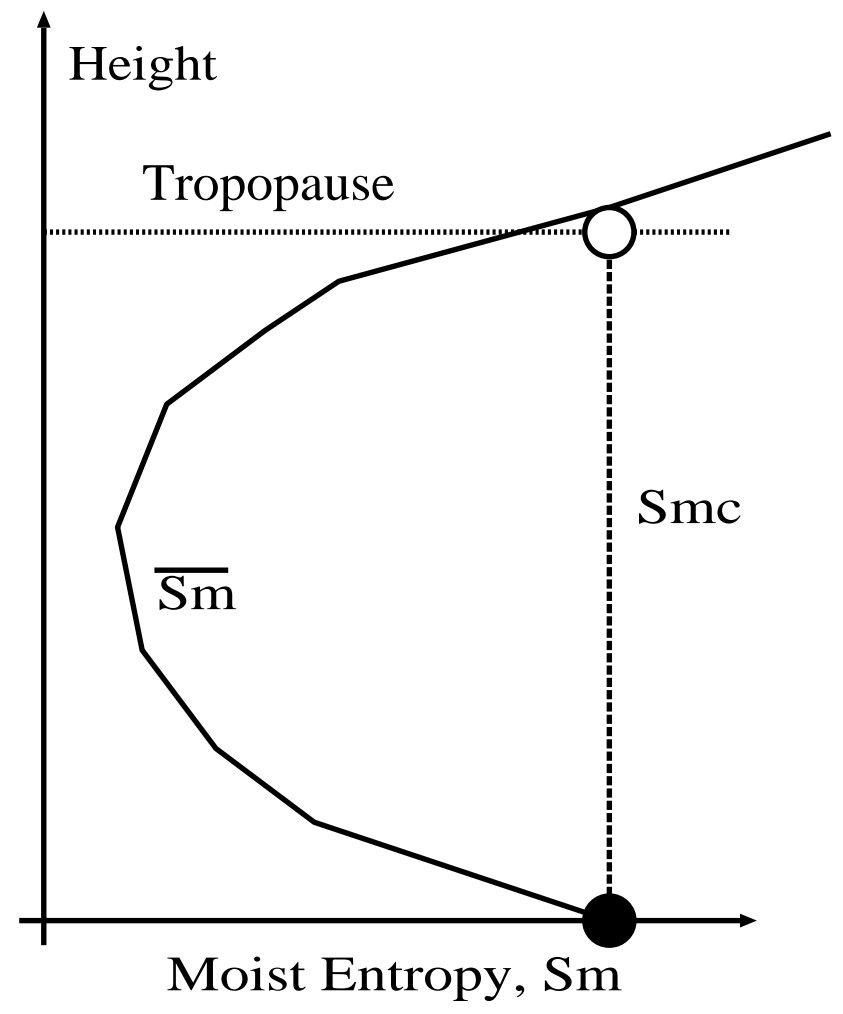

ACPD

9, 3535-3553, 2009

What is mass flux?

J.-I. Yano

Title Page

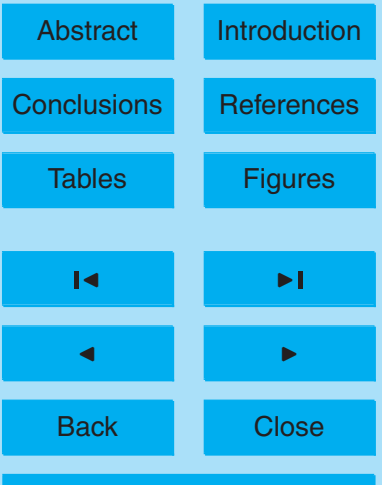

Full Screen / Esc

Fig. 1. A schematics of a typical vertical profile $\bar{S}_{m}$ (solid curve) of the moist entropy in the

Printer-friendly Version tropical atmosphere: it decreases with height up to a middle troposphere, then it turns to increase with height. Also shown is a possible profile $S_{m c}$ (dashed line) for a "hot tower". The Interactive Discussion entraining and the detraining levels of the hot tower are indicated by a closed and an open circles, respectively. 


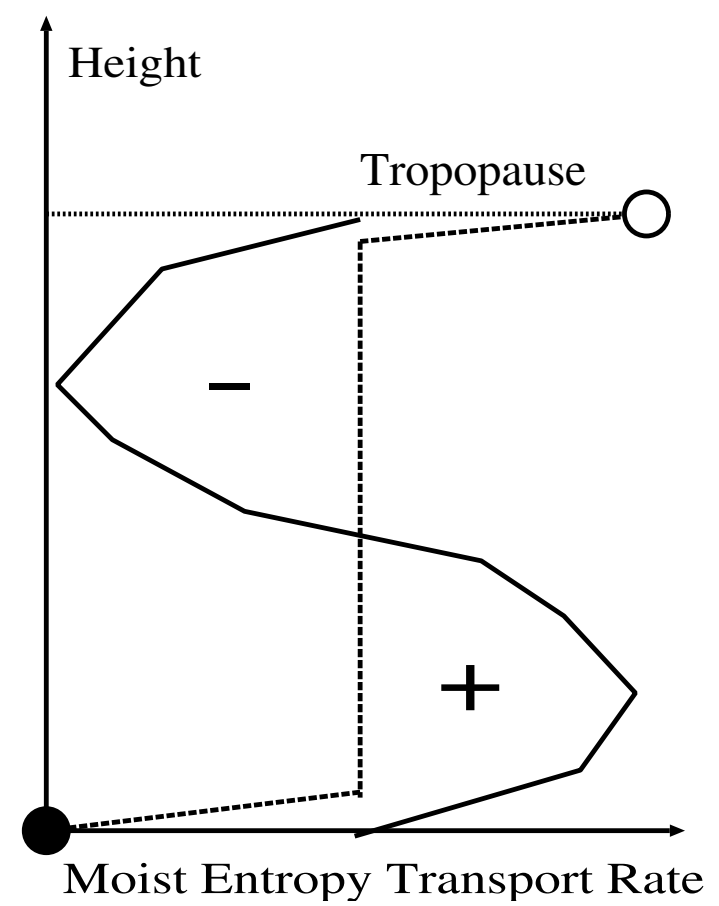

Fig. 2. Schematic curves for vertical transport rates of the moist entropy as functions of height with two given vertical profiles in Fig. 1 under a vertical ascent. The climatological profile $\bar{S}_{m}$ (solid curve in Fig. 1) gives upwards (positive) and downwards (negative) transports in lower half and upper half troposphere(solid curve). On the other hand, a vertically-homogeneous profile $S_{m c}$ (dashed curve in Fig. 1) provides a sharp sink and source at the surface and the tropopause levels, respectively (dashed curve) as shown by Eq. (11) below. Those sink and source balance with the surface flux and radiative cooling, respectively, thus the entropy budget is closed.

\section{ACPD}

9, 3535-3553, 2009

What is mass flux?

J.-I. Yano

Title Page

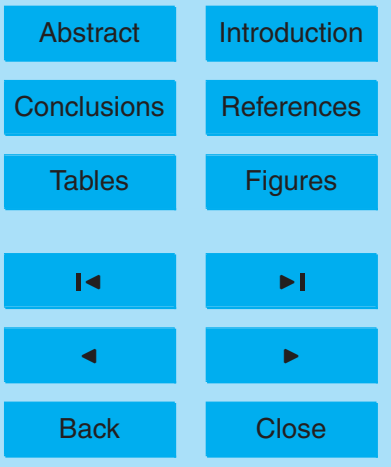

Full Screen / Esc

Printer-friendly Version

Interactive Discussion 


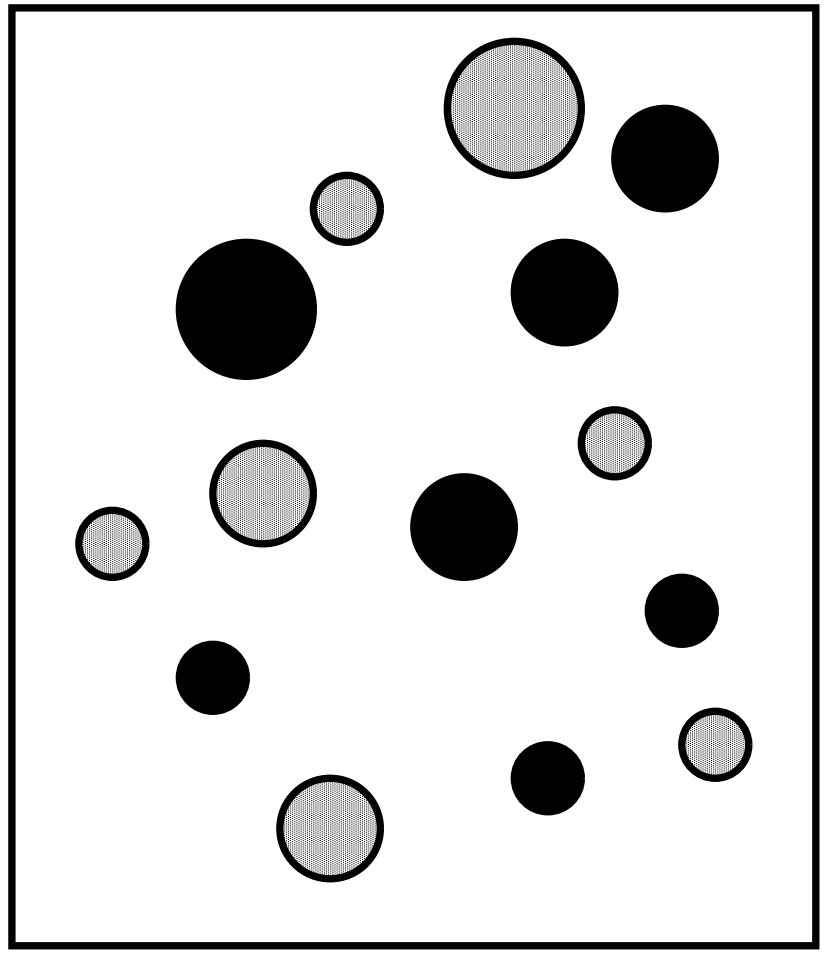

9, 3535-3553, 2009

What is mass flux?

J.-I. Yano

Title Page

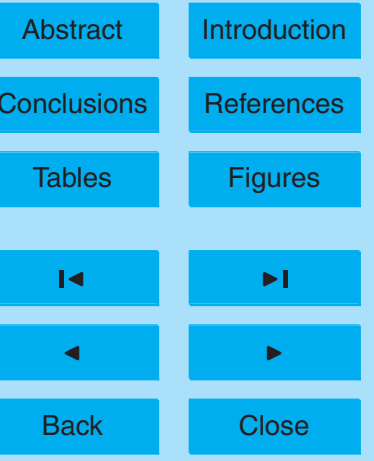

Full Screen / Esc

Printer-friendly Version

Fig. 3. A schematic representing a distribution of convective updrafts (dark circle) and downdraft (grey circles) within a square horizontal box (top view). The remaining area (shown in white) within the square box corresponds to the environment.

Interactive Discussion

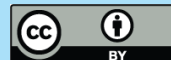

